\title{
PERFORMANCE OF AN ADAPTIVE CONTROLLER FOR THE NEUROMUSCULAR BLOCKADE BASED ON INVERSION OF A WIENER MODEL
}

\author{
M. M. Silva, L. Paz, T. Wigren, and T. Mendonça
}

\begin{abstract}
An adaptive controller based on a minimally parameterized parsimonious Wiener model for the effect of the muscle relaxant rocuronium in the neuromuscular blockade is presented. The controller structure combines inversion of the recursively identified static nonlinearity of the Wiener model with a positive compartmental control law for the linearized system. The overall strategy exploits the fact that the model has only two parameters, which are estimated by an extended Kalman filter. Due to the fact that the positive control law for total mass conservation of compartmental systems is only proven to be convergent for time-invariant systems, the identification of the parameter in the linear block of the minimally parameterized parsimonious Wiener model is stopped when the controller is turned on. The controller was implemented in the platform Galeno and tested in simulation and in thirteen real cases of patients under general anesthesia. The good reference tracking results and robustness to noise show the reliability of the proposed strategy.
\end{abstract}

Key Words: Anesthesia, compartmental models, drug delivery systems, minimally parameterized models, neuromuscular blockade, Wiener systems.

\section{INTRODUCTION}

During general anesthesia, depression of neuromuscular function is often required to intubate the patient and to enable easy access to the patient's internal organs. The conventional procedure to provide muscle relaxation is the manual administration of bolus doses of muscle relaxants.

With the exception of the initial bolus, the size of which is calibrated according to the patient's weight, the sizes of subsequent doses are usually based on the anesthesiologist's experience. This procedure gives considerable fluctuations in the levels of relaxation [1]. Moreover, since most of the muscle relaxants have high therapeutic indices in hospital settings, and due to the lack of a priori

M. M. Silva, L. Paz and T. Mendonça are with the Departamento de Matemática, Faculdade de Ciências, Universidade do Porto, Rua do Campo Alegre, 4169-007 Porto, Portugal.

M. M. Silva, and T. Mendonça are also with CIDMA, Campus Universitário de Santiago, 3810-193 Aveiro, Portugal.

M. M. Silva (corresponding author, e-mail: margarida silva@fc.up.pt) and T. Wigren are with the Division of Systems and Control, Department of Information Technology, Uppsala University, Box 337, SE-751 05 Uppsala, Sweden knowledge of the drug-patient interaction, they are often used in excess of their minimal effective requirements [2]. This overdosing eliminates fine control of the neuromuscular blockade (NMB) and may increase the incidence of side-effects. In this context, automatic closed-loop control of muscle relaxant administration appears to be a beneficial option. The advantages are twofold. Besides avoiding overdosing, the achievement of a better regulation of the NMB also enables a more meaningful evaluation of patient's depth of anesthesia [3]. However, in spite of the relatively high number of available automatic control strategies for the administration of muscle relaxants [4-6], they are still not popular in day-to-day clinical practice.

The muscle relaxant that is nowadays most extensively used in clinical practice is rocuronium. The main reason for this is the release of Sugammadex, the first selective rocuronium binding agent which enables a fast and total recovery of the neuromuscular function [7]. Despite the numerous advantages [8], the use of Sugammadex in the daily routine is still limited due to its high cost. Consequently, if fine control of the NMB is maintained by automatic controllers, rapid recovery of the neuromuscular function at the end of the surgery is more easily attained and Sugammadex can be saved for emergency situations or when standard reversal is contraindicated. Additionally, eye surgeries, or any others where deep internal organs have to be accessed, require 
a highly accurate control of the NMB around a setpoint. This accurate regulation is time-consuming and difficult to achieve by manual bolus administration protocols, or by manual titration of the individualized amount of muscle relaxants to be administered in a continuous intravenous infusion. These facts justify the development of closed-loop control strategies for the administration of rocuronium to patients under anesthesia.

Several quantitative methods based on the assessment of an evoked response to a pattern of stimulation of a motor nerve are commercially available to monitor the NMB [9]. The choice of the stimulation pattern is mostly correlated with the type of surgery and/or with the level of accuracy that is needed. A train-of-four (TOF) electrical stimulation of a peripheral muscle (e.g. the adductor pollicis in the hand of the patient) is a method commonly adopted in studies with closed-loop NMB control [10-12] due to its ease of use.

According to [13, pp. 544], applying a TOF stimulation at $2 \mathrm{~Hz}$ provides more sensitivity than a single twitch and approximately the same sensitivity as tetanic stimulation at $50 \mathrm{~Hz}$. Moreover, the relatively low frequency of this stimulation pattern allows the response to be evaluated manually or visually, which is of crucial importance in the clinical setting where the anesthesiologist has a supervisory role.

The main contributions of this paper follow from the fact that, to the authors' knowledge, no closed-loop control strategy using the first twitch of a TOF stimulation as the output signal to quantify the effect of rocuronium in patients under general anesthesia has consistently shown good performance in clinical conditions. The structure of the new controller comprises online identification of two parameters in a Wiener model, online inversion of the nonlinearity at each time step using the online identified nonlinear parameter, and linear control, this structure being the first contribution of the paper. The linear control relies on a compartmental control law that aims at keeping the total mass of the system (i.e. the total mass of rocuronium in the patient) constant. Crucial for the good performance of the whole strategy is the fact that the compartmental control law depends explicitly only on the linear parameter of the Wiener model. A further advantage is that the compartmental control algorithm naturally copes with the presence of positive constraints, and is proven to be robust to parameter uncertainties [14]. The second contribution of the paper concerns the implementation of the controller in the platform Galeno [15], and its performance evaluation on patients under general anesthesia.

This paper is organized as follows. Section II describes the two-parameters minimally parameterized parsimonious (MPP) model used for the system mod- eling and the recursive identification strategy exploiting the extended Kalman filter (EKF). Section III presents the main features of the closed-loop control strategy while section IV shows the results. Section V draws the conclusions.

\section{THE MODEL AND THE IDENTIFICATION ALGORITHM}

\subsection{The MPP Wiener model for the NMB}

This section presents the MPP Wiener model describing the effect of the muscle relaxant rocuronim in the human body. This model was initially developed for atracurium [16] and further validated for rocuronium [17]. The same minimal modeling principle was also successfully applied to explain the effect of hypnotics and analgesics in the depth of anesthesia [18,19] and used for the development of model-based controllers, see e.g. [20].

A block diagram of the MPP Wiener model for the effect of muscle relaxants in the NMB is shown in Fig. 1. The model input is the rocuronium rate $u(t)(\mu \mathrm{g} / \mathrm{kg} / \mathrm{min})$ that is administered to the patient, and the model output is the NMB level $y(t)(\%)$, normalized between 0 (corresponding to full paralysis) and 100 (corresponding to full muscular activity) due to the fact that, in this paper, the NMB level is considered to be the first TOF response calibrated by a reference twitch. The transfer function of the linear dynamic part of the Wiener model is given by

$$
G_{p}(\alpha)=\frac{k_{1} k_{2} k_{3} \alpha^{3}}{\left(s+k_{1} \alpha\right)\left(s+k_{2} \alpha\right)\left(s+k_{3} \alpha\right)},
$$

which may be realized in state-space form as

$$
\begin{aligned}
& \left\{\begin{array}{l}
\dot{x}(t)=A(\alpha) x(t)+B(\alpha) u(t), \\
y_{l}(t)=C x(t)
\end{array}\right. \\
& A(\alpha)=\left[\begin{array}{ccc}
-k_{3} \alpha & 0 & 0 \\
k_{2} \alpha & -k_{2} \alpha & 0 \\
0 & k_{1} \alpha & -k_{1} \alpha
\end{array}\right], \\
& B(\alpha)=\left[\begin{array}{lll}
k_{3} \alpha & 0
\end{array}\right]^{T}, \\
& C=\left[\begin{array}{lll}
0 & 0 & 1
\end{array}\right] . \\
& \stackrel{u}{\longrightarrow} G_{p}(\alpha) \stackrel{y_{l}}{\longrightarrow} \underset{r\left(\gamma, y_{l}\right)}{\longrightarrow}
\end{aligned}
$$

Fig. 1. Block diagram of the MPP Wiener model for the effect of muscle relaxants in the NMB. 
The constants $k_{1}=1, k_{2}=4$, and $k_{3}=10$ are fixed $[16,18]$, and the identified parameter $\alpha>0$ reflects the inter-patients' variability.

The state-space model (2a) with the system matrices defined as (2b)-(2d) was shown to be a compartmental model [21] in [22].

The nonlinear part of the MPP Wiener model relates the output $y_{l}(t)$ of the linear block with the NMB level $y(t)$ through the Hill function [23] as

$$
\begin{aligned}
r:\left(\gamma, y_{l}(t)\right) & \in] 0,+\infty[\times[0,+\infty[ \\
& \left.\left.\longmapsto y(t)=r\left(\gamma, y_{l}(t)\right) \in\right] 0,100\right],
\end{aligned}
$$

where

$$
r\left(\gamma, y_{l}(t)\right)=\frac{100 C_{50}^{\gamma}}{C_{50}^{\gamma}+\left(y_{l}(t)\right)^{\gamma}} .
$$

The parameter $\gamma>0$ describes the sigmoidicity of the Hill function and it is the one to be identified in the nonlinear block of the Wiener model. The parameter $C_{50}$ is fixed and equal to 1 [17].

In order to implement the model and the controller in the platform Galeno [15] for simulation and clinical trials, the continuous-time model (2), (3) has to be sampled using a zero-order hold method [24]. The discrete-time model becomes

$$
\begin{aligned}
& \left\{\begin{array}{c}
x(k h+h)=A_{d}(\alpha) x(k h)+B_{d}(\alpha) u(k h), \\
y_{l}(k h)=C x(k h)
\end{array}\right. \\
& A_{d}(\alpha)=e^{A(\alpha) h}, \\
& B_{d}(\alpha)=\int_{0}^{h} e^{A(\alpha) s} d s B(\alpha),
\end{aligned}
$$

where $u(k h)$ is the input (piecewise constant rocuronium rate); $x(k h)$ is the discrete-time state-vector; $y_{l}(k h)$ is the discrete-time output of the linear block; and $A_{d}(\alpha)$ and $B_{d}(\alpha)$ are the sampled system matrices.

Due to the fact that in the surgery room data from the NMB is monitored and acquired every 20 seconds to ensure that all the nerve fibers are recruited every time an electrical stimulation is performed, the zero-order hold method [24] is applied using $h=1 / 3 \mathrm{~min}^{-1}$.

Since the sampling does not affect the nonlinear part, (3) can be used as it is and the model output becomes

$$
y(k h)=r\left(\gamma, y_{l}(k h)=\frac{100 C_{50}^{\gamma}}{C_{50}^{\gamma}+\left(y_{l}(k h)\right)^{\gamma}} .\right.
$$

\subsection{The extended Kalman filter}

Considering the model (1),(3), the parameter vector to be identified in the EKF structure is selected as

$$
\theta=\left[\begin{array}{ll}
\alpha & \gamma
\end{array}\right]^{T} .
$$

Since the linear block (1) was chosen to contribute with a unity gain for the whole system, the gain of the whole system must be estimated by the parameter $\gamma$ in (3). This is so because, in a Wiener model structure, only the product of the static gains of the two cascaded blocks is important from an input-output point of view [25]. At the same time, $\gamma$ adapts the shape or nonlinear static differential gain of (3).

To describe the EKF, the underlying general discrete-time nonlinear model is assumed to be

$$
\begin{aligned}
\hat{x}(k h+h)= & f(k h, \hat{x}(k h), u(k h))+ \\
& +g(k h, \hat{x}(k h)) v(k h) \\
\hat{y}(k h)= & h(k h, \hat{x}(k h))+e(k h),
\end{aligned}
$$

where $v(k h)$ and $e(k h)$ are mutually independent Gaussian white noise sequences with zero means and covariances $R_{1}(k h)$ and $R_{2}(k h)$, respectively. The EKF algorithm can then be summarized as follows (see e.g. [26]):

$$
\begin{aligned}
H(k h)= & \left.\frac{\partial h(k h, x)}{\partial x}\right|_{x=\hat{x}(k h \mid k h-h)} \\
K(k h)= & P(k h \mid k h-h) H^{T}(k h) \times \\
& \times\left[H(k h) P(k h \mid k h-h) H^{T}(k h)+R_{2}(k h)\right]^{-1} \\
\hat{x}(k h \mid k h)= & \hat{x}(k h \mid k h-h)+ \\
& +K(k h)[y(k h)-h(k h, \hat{x}(k h \mid k h-h))] \\
P(k h \mid k h)= & (k h \mid k h-h)-K(k h) H(k h) P(k h \mid k h-h) \\
\hat{x}(k h+h \mid k h)= & f(k h, \hat{x}(k h \mid k h), u(k h)) \\
F(k h)= & \left.\frac{\partial f(k h, x)}{\partial x}\right|_{x=\hat{x}(k h \mid k h)} \\
G(k h)= & \left.g(k h, x)\right|_{x=\hat{x}(k h \mid k h)} \\
P(k h+h \mid k h)= & F(k h) P(k h \mid k h) F^{T}(k h)+ \\
& +G(k h) R_{1}(k h) G^{T}(k h)
\end{aligned}
$$

The dependency of the state vector $x$ on $\alpha$ is not explicitly represented here for the sake of notational simplicity.

To enable the estimation of the model parameters with the EKF, a coupled identification model that merges the sampled model (4) with a random walk model [27] for 
the parameter estimates of (6) is defined. The resulting augmented state vector $\bar{x}$ becomes

$$
\bar{x}(k h)=\left[\begin{array}{lllll}
x_{1}(k h) & x_{2}(k h) & x_{3}(k h) & \alpha(k h) & \gamma(k h)
\end{array}\right]^{T} .
$$

Using (9), the extended state-space model becomes

$$
\begin{aligned}
& \hat{\bar{x}}(k h+h)=\left[\begin{array}{cc}
A_{d}(\hat{\alpha}(k h)) & \underline{0}_{3 \times 2} \\
\underline{0}_{2 \times 3} & I
\end{array}\right]\left[\begin{array}{l}
\hat{x}(k h) \\
\hat{\alpha}(k h) \\
\hat{\gamma}(k h)
\end{array}\right]+ \\
& +\left[\begin{array}{c}
B_{d}(\hat{\alpha}(k h)) \\
\underline{0}_{2 \times 1}
\end{array}\right] u(k h)+\left[\begin{array}{c}
v_{x}(k h) \\
v_{\alpha}(k h) \\
v_{\gamma}(k h)
\end{array}\right] \\
& \equiv\left[\begin{array}{c}
f_{1}(k h, \hat{\bar{x}}(k h), u(k h)) \\
\vdots \\
f_{5}(k h, \hat{\bar{x}}(k h), u(k h))
\end{array}\right]+v(k h) \\
& \equiv f(k h, \hat{\bar{x}}(k h), u(k h))+v(k h), \\
& \hat{y}(k h)=\frac{100 C_{50}^{\hat{\gamma}(k h)}}{C_{50}^{\hat{\gamma}(k h)}+(\bar{C} \hat{\bar{x}}(k h))^{\hat{\gamma}(k h)}}+e(k h) \\
& \equiv h(k h, \hat{\bar{x}}(k h))+e(k h) \text {, } \\
& \bar{C}(\cdot)=\left[\begin{array}{lll}
C(\cdot) & 0 & 0
\end{array}\right] .
\end{aligned}
$$

In the EKF algorithm structure (8) both $f$ and $h$ have to be linearized. The linearization of $f(k h, \hat{\bar{x}}(k h), u(k h))$ in (10) was performed analytically. The formula for $F(k h)$ is not shown here due to its complexity. In order to reduce the computational complexity of the calculations, the linearization of $h(k h, \hat{\bar{x}}(k h))$ in (11) was performed numerically as

$$
H(k h)=\frac{h(k h, \hat{\bar{x}}(k h)+\Delta \hat{\bar{x}}(k h))-h(k h, \hat{\bar{x}}(k h))}{\Delta \hat{\bar{x}}(k h)},
$$

where $\Delta \hat{\bar{x}}(k h)$ is the step for the differentiation and is chosen to be small.

Although not the same estimation algorithm as in [25], the use of a Wiener model implies that convergence requires that the input signal is sufficiently rich in frequencies and amplitude [25]. A theoretical analysis of the EKF in the setting used here would be a substantial effort and is a suitable topic for future research.

\section{THE NONLINEAR ADAPTIVE CONTROLLER}

\subsection{Induction and initialization: practical aspects}

In order to enable the patient's intubation, the induction of the NMB in patients under anesthesia must be quick. For this purpose, and due to clinical constraints, a manual bolus with a typical value of $600 \mu \mathrm{g} / \mathrm{kg}$ of rocuronium is usually given to the patient at the beginning of the surgery. This value may change depending on the requirements of the surgery and the patient's general clinical state.

Due to the fact that, in this paper, the NMB is quantified by the first response from a TOF stimulation normalized by a reference twitch, before the bolus administration the NMB is constant and equal to $100 \%$, independently of the patient or patient model in question. Hence the time of the bolus administration is defined in this paper as $t=0$ because it is only after this instant that informative data about the patient specific response to the muscle relaxant is collected.

In typical cases, an initial bolus of $600 \mu \mathrm{g} / \mathrm{kg}$ of rocuronium causes a decrease in the NMB from $100 \%$ to a value close to $0 \%$ in less than 4 minutes and is expected to provide between 15 and 85 minutes of muscle relaxation under opioid/nitrous oxide/oxygen anesthesia [28]. Due to this high variability in the duration of the clinical effect, an OnLine tuned Algorithm for Recovery Detection (OLARD) [29] is used to detect the beginning of the recovery after the initial bolus, this time being defined as $t_{0}$. At $t=t_{0}$ the controller is turned on.

Since the positive control law for total mass conservation of compartmental systems is only proven to be convergent for time-invariant systems, the identification of the linear parameter of the MPP Wiener model is stopped when the controller is turned on (i.e. for $t \geq t_{0}$ ). Hence, for $t \geq t_{0}$, the time-varying behavior of the system is solely captured by the estimation of the nonlinear parameter $\gamma$. This improves identifiability further, reducing the number of parameters estimated online to one.

\subsection{Structure}

The structure of the adaptive controller proposed in this paper is shown in Fig. 2 and comprises three main tasks that are performed in order at each time step.

First, using the measured input $u$, and output $y$ from the patient (i.e. the plant), the EKF block in Fig. 2 identifies the model parameters in (6) online.

Second, the target reference value $y^{*}$ for the NMB is inverted through the static nonlinearity (5) using the current estimate $\hat{\gamma}$ of the nonlinear parameter. This step 


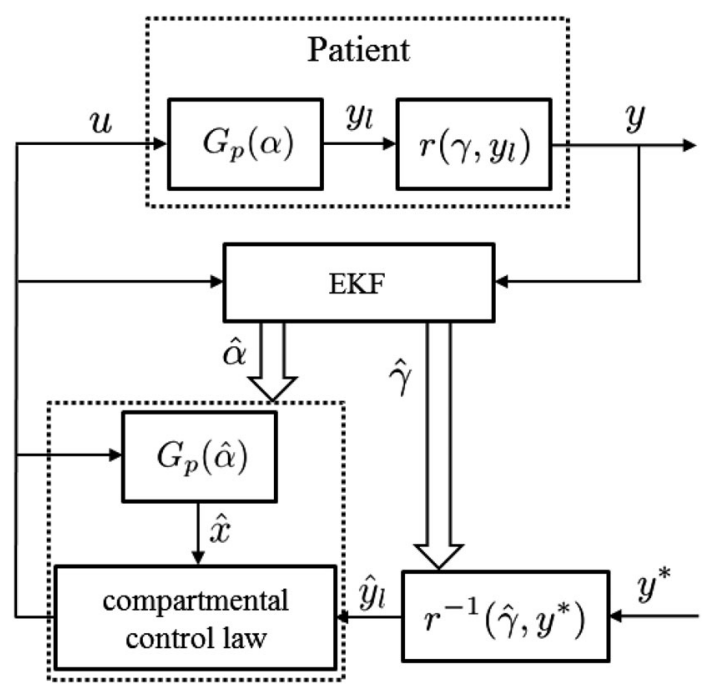

Fig. 2. Adaptive controller based on the minimally parameterized Wiener model for the NMB.

provides the compartmental control law with an estimate of the intermediate signal $\hat{y}_{l}$ in the Wiener model to be used for the calculation of the target total mass $M^{*}$ of the system (see (17) and recall that, from (2a) and (2d), $\left.y_{l}(t)=x_{3}(t)\right)$.

Third, a compartmental linear control law is applied to the estimated plant. For that, an estimate of the states of the compartmental model (2a) are obtained via simulation of the linear block of the Wiener model, using the estimate of the linear parameter $\alpha$.

\subsection{Inversion of the nonlinearity in the Wiener model}

The idea of inverting the nonlinearity in the Wiener model derives from the structure proposed in [30]. In this paper, however, only the reference value $y^{*}$ is inverted through $r^{-1}$ (3) using the current estimate of $\gamma$ provided by the EKF identification block. The advantages of performing this inversion, especially in cases where the slope of the nonlinearity varies considerably depending on the operating point, are clearly exemplified in [31]. Instead of obtaining an estimate of the intermediate signal of the Wiener model by inverting the measured signal $y$, the estimate of $\alpha$ is used to generate an estimate of the intermediate signal $y_{l}$ via (1), as shown in Fig. 2.

It should be stressed that $r(3)$ is a bijective function, and that $y^{*}$ in Fig. 2 lays inside $[0,100]$ as a consequence of monitoring restrictions of the NMB in the clinical practice. Moreover, as a result of a projection algorithm in the EKF structure, $\hat{\gamma}$ is also lower-bounded. Due to this, and considering the domains affecting the Hill function (3), no problems arise in this inversion.

\subsection{Linear controller design}

The compartmental law for the stabilization of the total mass of a continuous-time system was proposed in [32]. When applied to the system (2), it becomes

$$
\begin{aligned}
& u(t)=\max (0, \tilde{u}(t)), \\
& \tilde{u}(t)=\frac{-\left(\left[\begin{array}{lll}
1 & 1 & 1
\end{array}\right] A_{c}(\alpha) x(t)+\lambda\left(M(x(t))-M^{\star}\right)\right)}{\left[\begin{array}{lll}
1 & 1 & 1
\end{array}\right] B_{c}(\alpha)},
\end{aligned}
$$

where $\lambda$ is a design parameter. This control law ensures the convergence of the total mass $M(x(t))$ of the system to a given set point $M^{\star}$. The total mass $M^{\star}$ of the system is given by

$$
M(x(t))=\sum_{i=1}^{3} x_{i}(t)
$$

where $x_{i}$ is the $i$ th element of the state vector $x$ [22].

The objective of the control law (13) is to steer the system to an equilibrium point that satisfies the two conditions:

$$
\begin{aligned}
& M(x(t))=M^{\star}, \\
& x_{1}(t)=x_{2}(t)=x_{3}(t) .
\end{aligned}
$$

Therefore, at the equilibrium point, the system satisfies (15) and (16). From (3), $M^{\star}$ can be written as

$$
M^{\star}=3 x_{3}(t)=3 r^{-1}\left(\gamma, y^{*}\right)=3\left(\frac{100}{y^{*}}-1\right)^{1 / \gamma} C_{50} .
$$

When the plant to be controlled is not known, estimates of the model parameters have to be used in the control law. This is the case of muscle relaxant administration to the human body. Given this, (17) becomes

$$
\hat{M}^{\star}=3 \hat{x}_{3}(t)=3 r^{-1}\left(\hat{\gamma}, y^{*}\right)=3\left(\frac{100}{y^{*}}-1\right)^{1 / \hat{\gamma}} C_{50} .
$$

The third component of the state-space vector $x_{3}(t)$ equals the intermediate signal $y_{l}(t)$ of the Wiener model (see (2)).

The transformation of the control law (13) into discrete-time was proposed in [33], and its complete 
mathematical realization may be found in [12]. The discrete-time positive compartmental control law is hence given by

$$
\begin{aligned}
& u(k h)=\max (0, \tilde{u}(k h)), \\
& \tilde{u}(k h)=\frac{\left[\begin{array}{lll}
1 & 1 & 1
\end{array}\right]\left(\lambda I-A_{d}(\alpha)\right) x(k h)+(1-\lambda) M^{\star}}{\left[\begin{array}{lll}
1 & 1 & 1
\end{array}\right] B_{d}(\alpha)} .
\end{aligned}
$$

\subsection{Implementation of the controller in the platform Galeno}

In order to assess its performance, the proposed adaptive controller was implemented in the platform

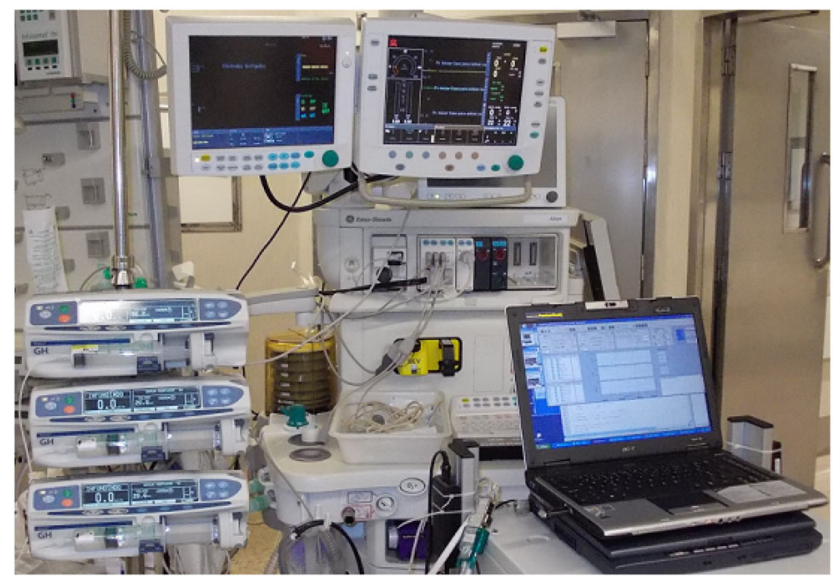

Fig. 3. Overview of the surgery room where the platform Galeno is being used.

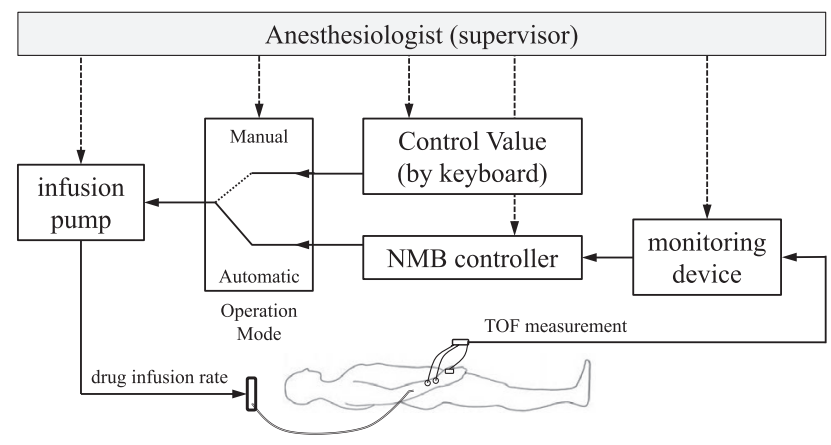

Fig. 4. Diagram of the NMB control functionality in the platform Galeno.
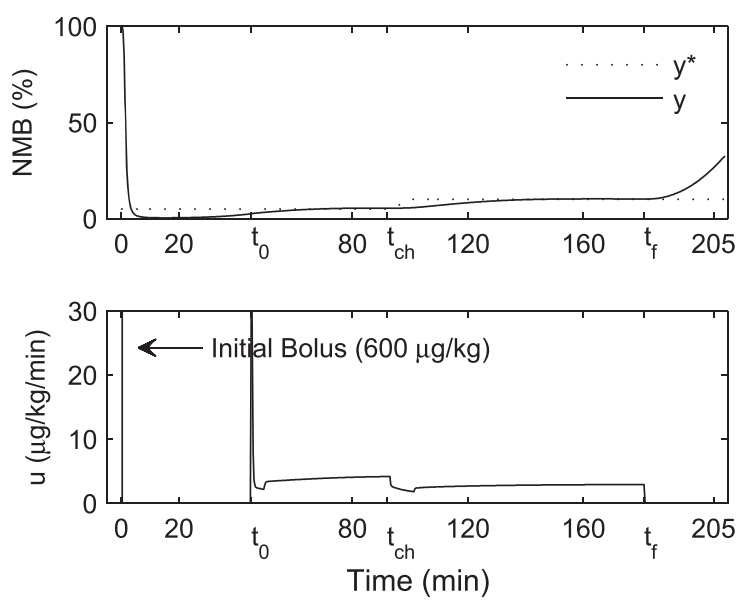

(a) Upper plot: controlled NMB $y$ and reference $y^{*}$. Bottom plot: control input (rocuronium infusion rate).
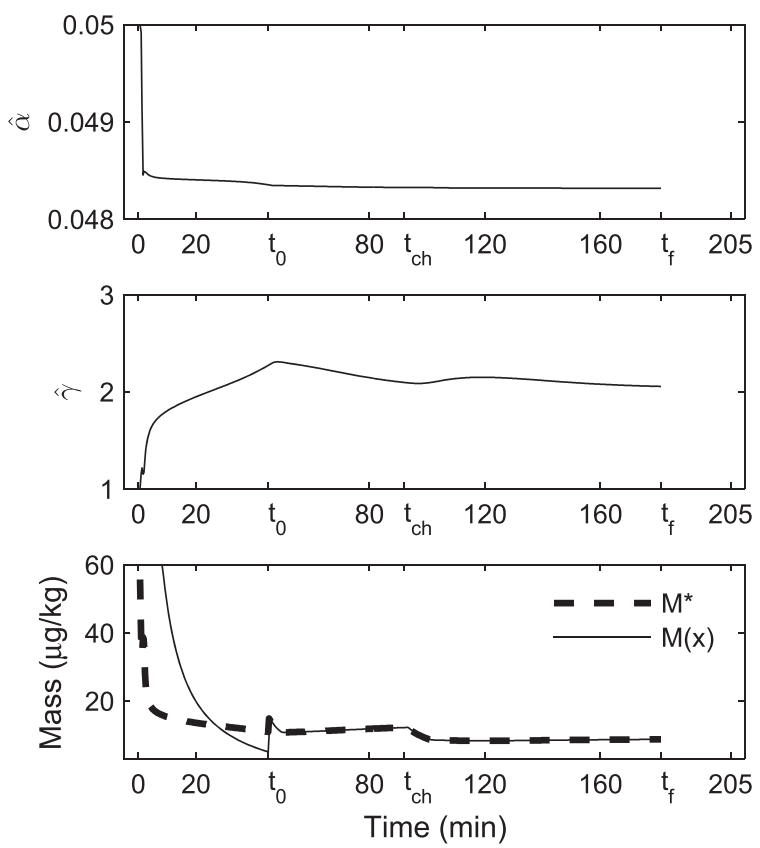

(b) Upper plot: estimates of parameter $\alpha$. Middle plot: estimates of parameter $\gamma$. Bottom plot: system mass $M(x)$ and reference mass $M^{*}$.

Fig. 5. Closed-loop control results in simulation.

Galeno [15]. Fig. 3 shows an overview of the surgery room where the platform Galeno is being used.

The platform architecture is structured in two application-components, the Galeno-Data Acquisition Drivers (GDAD) and the Galeno-Monitoring and Control of Anesthesia (GMCA). The GDAD were developed using the programming language $\mathrm{C \#}$ and are used only in real-time mode, with the main purpose of implementing an abstraction of the communication between the GMCA and the different syringe pumps and monitoring 
devices. The GMCA application was developed using Matlab (Version 7.9.0.529, R2009b, win32) and it may be used in real-time mode or in off-line mode. The main motivation for using MATLAB to implement GMCA is that it allows fast software prototyping and it facilitates the access to mathematical functions and methods that are necessary to implement the identification and control algorithms.

The NMB control functionality is part of the GMCA and is schematically represented in Fig. 4. Considering the recommendations of the anesthesiology team involved in the 'project Galeno', the following sequence of procedures is performed by order at the beginning of each general anesthesia episode:

1. Calibration of the NMB sensor.

2. Administration of the first bolus of rocuronium.

3. Detection of the time when the recovery after the first bolus starts, using the OLARD [29].

4. Start of the control algorithm (Automatic Operation Mode in Fig. 4). The NMB reference (Control Value in Fig. 4) is selected manually by the anesthesiologist.

\section{RESULTS}

\subsection{Simulation}

Fig. 5 shows the results of the adaptive controller when applied in simulation.

As shown in the bottom plot of Fig. 5a, the initial rocuronium bolus dose of $600 \mu \mathrm{g} / \mathrm{kg}$ led to a drop of the NMB level from $100 \%$ to $0 \%$ in a short period of time. After this initial bolus, no drug was administered until the recovery from this initial bolus was detected, being this time instant defined as $t_{0}$. From $t_{0}$ to $t_{c h}$ the reference was set to $5 \%$, and at time $t_{c h}$ changed to $10 \%$. As shown in the upper plot of Fig. 5a, the controlled NMB $y$ follows the reference $y^{*}$ with no static error. The behavior of the administered drug dose $u$, shown in the bottom plot of Fig. 5a is as desired: an impulse around time $t_{0}$ and an almost constant dose afterwards, until $t_{f}$ when the infusion is stopped and the system recovers naturally. The change that occurs in the dose profile at $t_{c h}$ is due to the change in the reference value. A good reference following is also observed with the total system mass $M(x)$, shown in the bottom plot of Fig. 5b. After $t_{0}$, the total system mass $M(x)$, in the solid line at the bottom plot of Fig. 5b is steered to the reference mass $M^{*}$, in dashed line in the bottom plot of Fig. 5b. The adaptivity of the control scheme is due to the adaptation of the model parameters, shown in the upper and middle plots of Fig. 5b. In the upper plot, the estimates of parameter $\alpha$ are represented. The convergence is fast at the very beginning and some changes exist until $t_{0}$. As expected, the value of $\alpha$ does not change significantly after $t_{0}$, and during the time when the controller is working. The adaptation that is needed to track the intra-patient's variability after $t_{0}$ is hence given only by the nonlinear parameter $\gamma$, represented in the middle plot of Fig. 5b. After the induction period, the parameter $\gamma$ presents values around 2 which is equivalent to a moderate steepness of the Hill function (3).

\subsection{Real cases}

The proposed adaptive NMB controller was tested in the surgery room in thirteen patients under general anesthesia, exhibiting a good clinical performance. The demographics of the patients are shown in Table I. The anesthetic protocols included combinations of propofol

Table I. Demographics of the patients. The ASA refers to the physical status classification system in [35].

\begin{tabular}{lcccccc}
\hline Case & Duration $(\mathrm{min})$ & ASA & Gender & Age (yrs) & Weight $(\mathrm{kg})$ & Height $(\mathrm{cm})$ \\
\hline 1 & 232 & 4 & M & 63 & 87 & 165 \\
2 & 120 & 2 & F & 63 & 76 & 160 \\
3 & 86 & 2 & F & 55 & 70 & 165 \\
4 & 400 & 2 & M & 51 & 64 & 165 \\
5 & 281 & 2 & F & 73 & 65 & 160 \\
6 & 249 & 2 & M & 69 & 60 & 165 \\
7 & 211 & 3 & F & 66 & 63 & 155 \\
8 & 133 & 2 & F & 28 & 76 & 169 \\
9 & 192 & 2 & F & 86 & 53 & 155 \\
10 & 206 & 3 & F & 77 & 65 & 157 \\
11 & 120 & 3 & M & 68 & 76 & 173 \\
12 & 166 & 3 & M & 60 & 61 & 164 \\
13 & 155 & 3 & F & 78 & 75 & 155 \\
\hline
\end{tabular}


as hypnotic; remifentanil, sufentanil or fentanil as analgesic; and an inhalation anesthetic.

According to [34], the overall performance of a closed-loop control system may be characterized on the basis of the performance error (PE) calculated as the weighted difference between the measured NMB $y_{t}$ and the reference $y_{t}^{*}$ as $\mathrm{PE}_{t}=100\left(y_{t}-y_{t}^{*}\right) / y_{t}^{*}$. The median performance error MDPE $=\operatorname{Median}\left\{P E_{t}\right\}$ is a measure of bias and reflects whether the measured values are systematically either above or bellow the reference value. The median absolute performance error MAPE $=$ Median $\left\{|P E|_{t}\right\}$ measures the inaccuracy of the control method. The Wobble $=$ Median $\left\{\left|P E_{t}-M D P E\right|\right\}$ measures the oscillatory behavior of the controller.

The Varvel performance measurements for each of the thirteen real cases considering $t=\left\{t_{0}, \ldots, t_{f}\right\}$ are presented in Table II. The MDPE values show that the controlled NMB signal is, on average, 5.68\% below the reference value, while the MAPE values show that the controller was, on average, $22.4 \%$ inaccurate. The transients are the main reasons for the MAPE and wobble values. One example for this is case number 13 as shown in Fig. 7. A detailed description of the closed-loop control results for case numbers 4 and 13 is given in sections 4.2.1-4.2.2.

It should be noted that the rocuronium doses that were administered in these cases (see $\bar{u}$ values in Table II) comply with the administration guidelines for continuous infusion during maintenance [28]. Under total intravenous anesthesia the recommended values are $4-16 \mu \mathrm{g} / \mathrm{kg} / \mathrm{min}$, with a reduction of $40 \%$ in cases where inhalation anesthetics, in particular isoflurance and enflurance, are used.
The last four columns in Table II show the spread of the parameter estimates for $t \geq t_{0}$.

\subsubsection{Example \#1}

Contrarily to the simulation case shown in section 4.1, the anesthesiologist in this case chose to administer an initial bolus of $300 \mu \mathrm{g} / \mathrm{kg}$ of rocuronium, as shown in the bottom plot of Fig. 6a. Due to this low bolus dose, the measured NMB, the solid line in the upper plot of Fig. $6 \mathrm{a}$, did not reach $0 \%$, and the recovery was detected earlier than in the simulation case, this time instant being marked with $t_{0}$ in the plots. In order to exemplify the performance of the adaptive controller, and without repercussions on the patient under anesthesia, the reference value was changed three times, at the times marked with $t_{c h}$. Initially, and as recommended for general anesthesia episodes, the reference was kept low, at a value of $2.5 \%$, and made to increase afterwards. As shown in the upper plot of Fig. 6a, after the beginning of the recovery, i.e. for $t>t_{0}$, the NMB $y$ was always steered to the reference $y^{*}$, with no significant over or under shoots. The measured signal is more noisy than in the simulation case due to the sensor in use. The administered rocuronium was as shown in the bottom plot of Fig. 6a. The bottom plot of Fig. $6 \mathrm{~b}$ shows the total mass $M(x)$ of the system following the time-varying reference $M^{*}$ for $t>t_{0}$. The change in the reference mass is due to the changes in the identified parameter $\gamma$, shown in the middle plot of Fig. 6b. Similarly to the simulation case, the estimates of the parameter $\alpha$ converge rapidly after the initial bolus and remain constant until the third change in the reference value, at time $t_{c h 3}$. At that time

Table II. Performance of the controller when applied to the patients in Table I, for $t>t_{0}$. The values in the columns marked with ${ }^{(*)}$ and ${ }^{(* *)}$ have dimensions $10^{-2}$ and $10^{-5}$, respectively. The $\sigma_{\theta}$ denotes the standard deviation of the parameter $\theta$.

\begin{tabular}{lcccccccc}
\hline Case & MDPE $(\%)$ & MAPE $(\%)$ & Wobble $(\%)$ & $\bar{u}(\mu / \mathrm{kg} / \mathrm{min})$ & $\overline{\hat{\alpha}}^{(*)}$ & $\sigma_{\hat{\alpha}}^{(* *)}$ & $\overline{\hat{\gamma}}$ & $\sigma_{\hat{\gamma}}$ \\
\hline 1 & -10.0 & 12.4 & 6.29 & 4.36 & 4.76 & 1.39 & 2.03 & 0.200 \\
2 & -8.57 & 28.7 & 29.6 & 4.21 & 5.31 & 4.87 & 2.60 & 0.279 \\
3 & -24.8 & 24.8 & 11.2 & 6.45 & 3.98 & 1.32 & 1.67 & 0.183 \\
4 & -6.28 & 9.47 & 7.40 & 4.92 & 4.91 & 17.2 & 1.55 & 0.146 \\
5 & -3.64 & 46.6 & 46.4 & 1.88 & 4.71 & 14.6 & 3.83 & 0.908 \\
6 & -15.2 & 16.2 & 9.44 & 4.39 & 3.36 & 1.66 & 1.74 & 0.236 \\
7 & -9.36 & 18.8 & 14.6 & 3.98 & 3.70 & 8.28 & 1.96 & 0.237 \\
8 & -10.4 & 22.7 & 25.6 & 6.29 & 4.97 & 6.30 & 1.95 & 0.327 \\
9 & 15.0 & 35.0 & 27.5 & 2.24 & 4.03 & 5.87 & 4.71 & 0.297 \\
10 & -1.49 & 15.0 & 14.8 & 3.87 & 4.08 & 6.34 & 1.96 & 0.185 \\
11 & 4.45 & 12.4 & 14.2 & 6.13 & 4.47 & 1.00 & 1.90 & 0.088 \\
12 & -6.70 & 26.0 & 25.5 & 6.81 & 3.41 & 7.49 & 1.49 & 0.281 \\
13 & 3.19 & 23.3 & 21.2 & 3.04 & 3.45 & 4.56 & 2.77 & 0.265 \\
Mean & -5.68 & 22.4 & 19.5 & 4.50 & 4.24 & 6.22 & 2.32 & 0.279 \\
\hline
\end{tabular}



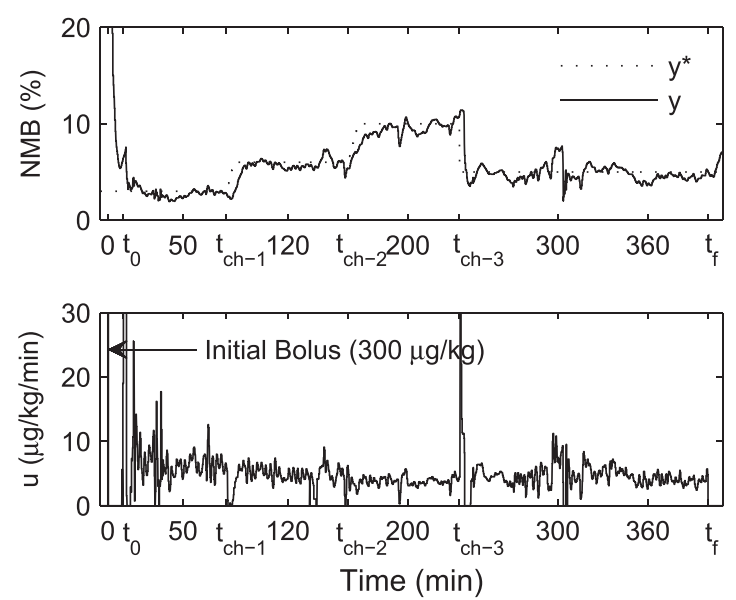

(a) Upper plot: controlled NMB $y$ and reference $y^{*}$. Bottom plot: control input (rocuronium infusion rate).
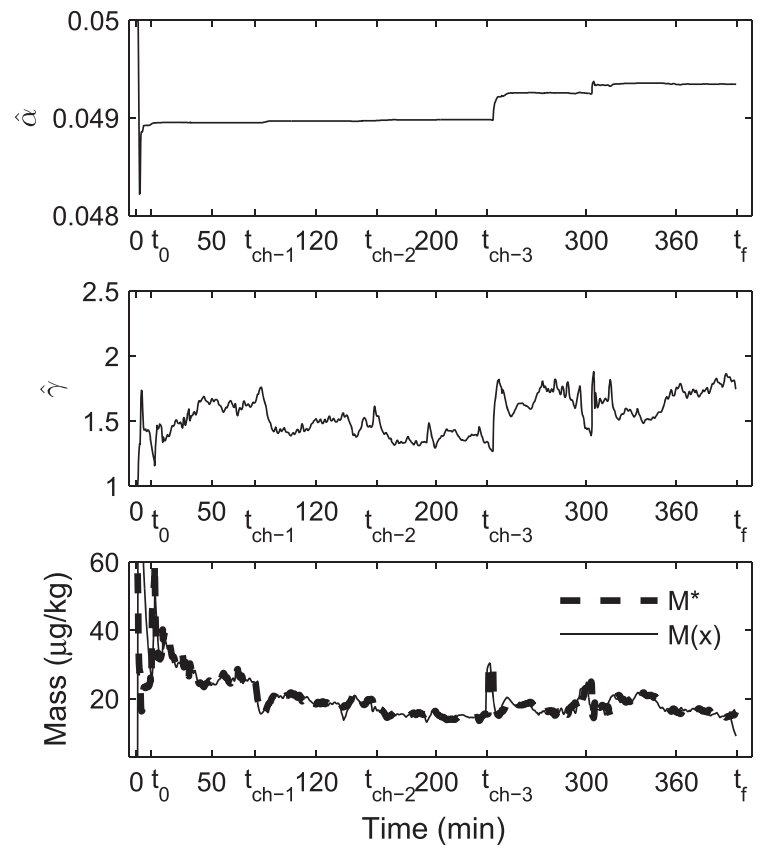

(b) Upper plot: estimates of parameter $\alpha$. Middle plot: estimates of parameter $\gamma$. Bottom plot: system mass $M(x)$ and reference mass $M^{*}$.

Fig. 6. Real closed-loop control results (Case number 4 in Table II).

instant, the parameter $\alpha$ changes slightly, with no negative effect on the controller performance in terms of reference tracking.

\subsubsection{Example \#2}

Fig. 7 shows the results obtained when using the proposed controller on patient number 13 in Table I and II. This is a typical case where the high MAPE is due
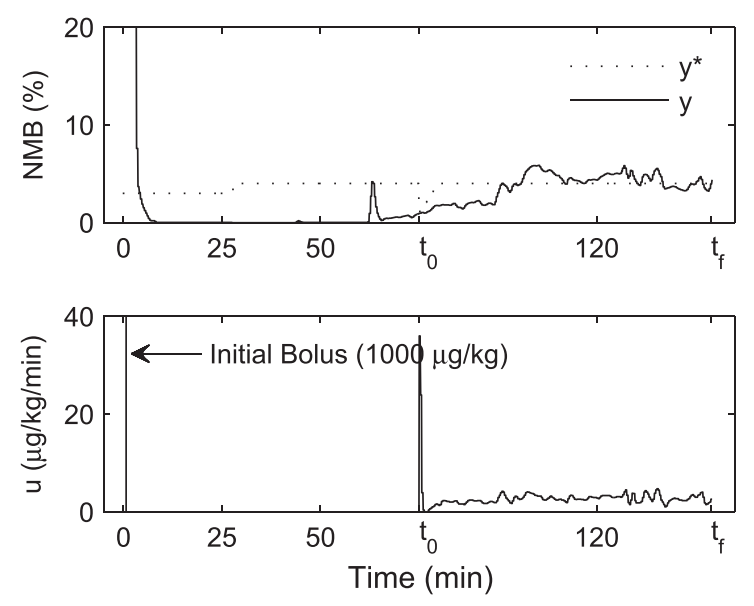

(a) Upper plot: controlled NMB $y$ and reference $y^{*}$. Bottom plot: control input (rocuronium infusion rate).
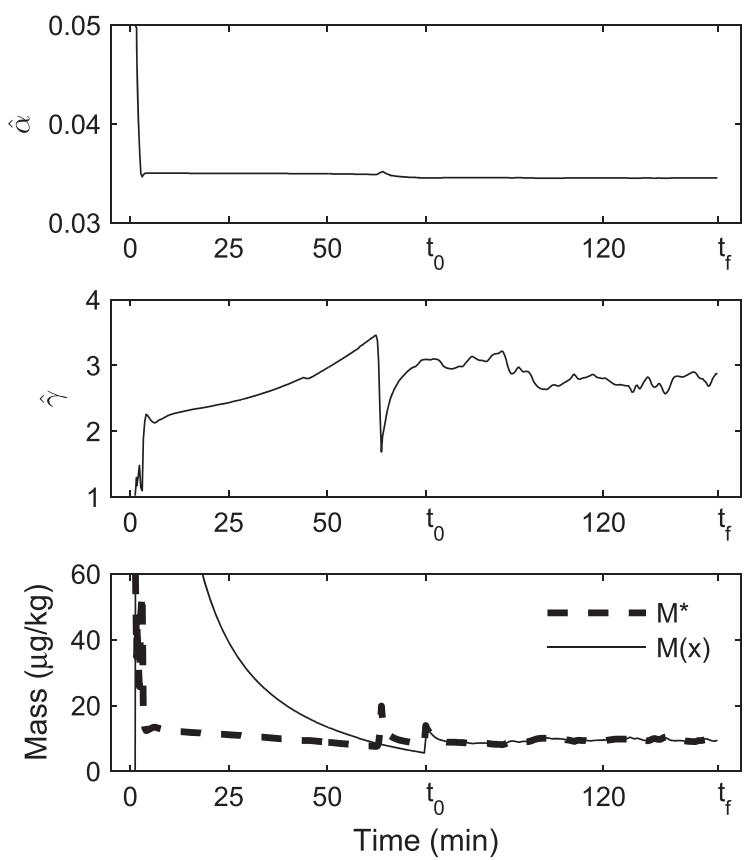

(b) Upper plot: estimates of parameter $\alpha$. Middle plot: estimates of parameter $\gamma$. Bottom plot: system mass $M(x)$ and reference mass $M^{*}$.

Fig. 7. Real closed-loop control results (Case number 13 in Table II).

to the difference between the measured $y$ and the reference $y^{*}$ right after the beginning of the infusion at $t=t_{0}$, as plotted in Fig. 7a. Clinically these closed-loop results are good, outperforming any manual infusion scheme. The administered rocuronium in shown in the bottom plot of Fig. 7a. The parameter estimates together with the system and reference mass are plotted in the upper and middle, and bottom plots of Fig. 7b, respectively. In 
Fig. $7 \mathrm{~b}$ it is clear that, for $t<t_{0}$, the model parameter estimates vary considerably, changing from their initial values at 0.005 and 1 , for $\alpha$ and $\gamma$ to values close to 0.035 and 3 , respectively. A consequence of this is a change in the total mass of the system, plotted in solid line in the bottom plot of Fig. $7 \mathrm{~b}$. For $t>t_{0}$, due to the parameters adaptation and the beginning of the closed-loop infusion, the total mass of the system $M(x)$ follows the target mass $M^{*}$, as desired.

\section{CONCLUSIONS}

This paper presented an adaptive controller for the NMB based on inversion of a Wiener model. The controller exploits a Wiener model with only two parameters, which are recursively estimated by an extended Kalman filter. The controller combines inversion of the recursively identified static nonlinearity of the Wiener model with a positive compartmental control law for the linearized system. With only the linear parameter explicitly involved, the complexity of the positive control law for total mass conservation of compartmental systems is significantly reduced, which is beneficial for the whole strategy.

This approach enhances the strategy in [12] since the identification of the nonlinear parameter is not pointwise, but recursive, which results in a more robust identification. The controller was implemented in the platform Galeno and tested in simulation and in real cases. As a consequence of the recursive identification of the model parameters, the variability of the patient parameters does not interfere with the performance of the controller. All tests showed a good reference tracking and robustness to noise. The good performance of the controller was also acknowledged by the medical staff that was present in the surgery room.

Interesting topics for future research include a convergence analysis of the recursive estimates and a stability analysis of the adaptive controller.

\section{REFERENCES}

1. Linkens, D. A., A. J. Asbury, S. J. Rimmer, and M. Menad, "Identification and control of muscle-relaxant anaesthesia," IEE Proc. Control Theory Applicat., Vol. 129, No. 4, pp. 136-141 (1982).

2. Gilhuly, T. J., G. A. Dumont, and B. A. MacLeod, "Modelling for computer controlled neuromuscular blockade," Proc. 27th Annual Intern. Conf. of the IEEE-EMBS, Shanghai, China, pp. 26-29 (2005).

3. Ekman, A., E. Stålberg, E. Sundman, L. I. Eriksson, L. Brudin, and R. Sandin, "The effect of neuromuscular block and noxious stimulation on hypnosis monitoring during sevoflurane anesthesia," Anesth. \& Analg., Vol. 105, No. 3, pp. 688-695 (2007).

4. Mendonça, T., H. Magalhães, P. Lago, and S. Esteves, "Hipocrates: a robust system for the control of neuromuscular blockade," J. Clin. Monitor. Comp., Vol. 18, pp. 265-273 (2004).

5. Eleveld, D. J., J. H. Proost, and J. M. K. H. Wierda, "Evaluation of a closed-loop muscle relaxation control system," Anesth. \& Analg., Vol. 101, pp. 758-764 (2005).

6. Gilhuly, T. J., B. A. MacLeod, G. A. Dumont, A. M. Bouzane, and S. K. W. Schwarz, "Improved neuromuscular blockade using a novel neuromuscular blockade advisory system: a randomized, controlled, clinical trial," Anesth. \& Analg., Vol. 107, No. 5, pp. 1609-1617 (2008).

7. Plaud, B., O. Meretoja, R. Hofmockel, J. Raft, P. A. Stoddart, J. H. M. van Kuijk, Y. Hermens, and R. K. Mirakhur, "Reversal of rocuronium-induced neuromuscular blockade with sugammadex in pediatric and adult surgical patients," Anesthesiology, Vol. 110, pp. 284-294 (2009).

8. Naguib, M., "Sugammadex: another milestone in clinical neuromuscular pharmacology," Anesth. \& Analg., Vol. 104, No. 3, pp. 575-581 (2007).

9. Hemmerling, T. M. and N. Le, "Brief review: Neuromuscular monitoring: an update for the clinician," Can. J. Anesth., Vol. 54, No. 1, pp. 58-72 (2007).

10. Mahfouf, M., D. A. Linkens, A. J. Asbury, W. M. Gray, and J. E. Peacock, "Generalised predictive control (GPC) in the operating theatre," IEE Proc-D, Vol. 139, No. 4, pp. 404-420 (1992).

11. Mendonça, T. and P. Lago, "PID control strategies for the automatic control of neuromuscular blockade," Control Eng. Practice, Vol. 6, No. 10, pp. 1225-1231 (1998).

12. Paz, L. A., M. M. Silva, S. Esteves, and T. Mendonça, "Self-calibrating total-mass controller for the neuromuscular blockade matching the anesthesiologists' mindset," Proc. 21st Mediterranean Conf. on Control Autom., Platanias-Chania, Crete, Greece, pp. 723-728 (2013).

13. Barash, P. G., Clinical Anesthesia, 7th edition, Wolters Kluwer/Lippincott Williams \& Wilkins, Hemel Hempstead, UK (2003).

14. Sousa, C., T. Mendonça, and P. Rocha, "Target mass control for uncertain compartmental systems," Int. J. Control, Vol. 83, No. 7, pp. 1387-1396 (2010).

15. Paz, L. A., J. Almeida, M. M. Silva, T. Mendonça, B. A. Costa, and S. Esteves, "Integrated design system for monitoring, digital processing and control in anesthesia," Proc. Amer. Soc. of Anesthe- 
siologists Annu. Meeting, Chicago, IL, USA, pp. A:1169 (2011).

16. Silva, M. M., T. Wigren, and T. Mendonça, "Nonlinear identification of a minimal neuromuscular blockade model in anesthesia," IEEE Trans. Control Syst. Technol., Vol. 20, No. 1, pp. 181-188 (2012).

17. Silva, M. M., R. Rabiço, T. Mendonça, and T. Wigren, "Control of rocuronium-induced neuromuscular blockade via online identification of a two-parameters Wiener model," Proc. 16th IFAC Symp. on Syst. Ident., Brussels, Belgium, pp. 571-576 (2012).

18. Silva, M. M., "Prediction error identification of minimally parameterized Wiener models in anesthesia," Proc. 18th IFAC World Congress, Milan, Italy, pp. 5615-5620 (2011).

19. Silva, M. M., T. Wigren, and T. Mendonça, "A reduced MIMO Wiener model for recursive identification of the depth of anesthesia," Int. J. Adapt. Control Signal Process. (2013). Early view - online at http://dx.doi.org/10.1002/acs.2447.

20. Nogueira, F. N., T. Mendonça, and P. Rocha, "Controlling the depth of anesthesia by a novel positive control strategy," Comp. Meth. Progr. Biomed., Vol. 114, No. 3, pp. e87 -e97 (2014).

21. Godfrey, K., Compartmental Models and their Application, Academic Press, London, UK (1983).

22. Almeida, J., M. M. Silva, T. Mendonça, and P. Rocha, "A compartmental model-based control strategy for neuromuscular blockade level," Proc. 18th IFAC World Congress, Milan, Italy, pp. 599-604 (2011).

23. Weatherley, B., S. Williams, and E. Neill, "Pharmacokinetics, pharmacodynamics and dose-response relationships of atracurium administered i. v.," Brit. J. Anaesth., Vol. 55, pp. 39s-45s (1983).

24. Åström, K. J. and B. Wittenmark, ComputerControlled Systems, Prentice Hall, Englewood Cliffs, NJ (1984).

25. Wigren, T., "Recursive prediction error identification method using the nonlinear Wiener model," Automatica, Vol. 29, No. 4, pp. 1011-1025 (1993).

26. Söderström, T., Discrete-Time Stochastic Systems, Springer-Verlag, London, UK (2002).

27. Söderström, T. and P. Stoica, System Identification, Prentice-Hall, Hemel Hempstead, UK (1989).

28. Zemuron, Zemuron (Rocuronium Bromide) Injection Solution for Intravenous Use, U.S. Food and Drug Administration, Initial U.S. Approval (1994).

29. Silva, M. M., C. Sousa, R. Sebastião, J. Gama, T. Mendonça, P. Rocha, and S. Esteves, "Total mass TCI driven by parametric estimation," Proc. 17th Mediterranean Conf. on Control and Autom., Thessaloniki, Greece, pp. 1149-1154 (2009).

30. Åström, K. J. and B. Wittenmark, Adaptive Control, 2nd ed., Dover Publications, Inc., Mineola, New York, USA (2008).

31. Wigren, T. and L. Brus, "Reduction of amplitude dependent gain variations in control of non-linear Wiener type systems," Proc. 7th IFAC Symp. on Nonlinear Control Syst., Pretoria, South Africa, pp. 730-735 (2007).

32. Bastin, G. and A. Provost, "Feedback stabilization with positive control of dissipative compartmental systems," Proc. 15th Int. Symp. on Math. Theory of Networks and Systems, Notre Dame, IN, USA, pp. 1-9 (2002).

33. Marques, E., "Sistemas Compartimentais," Master's Thesis, Aveiro, Portugal (2008).

34. Varvel, J. R., D. L. Donoho, and S. L. Shafer, "Measuring the predictive performance of computercontrolled infusion pumps," J. Pharmacokinet. Biop., Vol. 20, pp. 63-94 (1992).

35. American Society of Anesthesiologists, ASA physical status classification system. (Accessed 4 February 2014.) Available from: http://www.asahq. org/For-Members/Clinical-Information/. 
